\title{
Preconditioned Iterative Methods for Super-Resolution Image Reconstruction with Multisensors
}

\author{
Michael K. Ng Kenton N. Sze \\ Department of Mathematics, The University of Hong Kong \\ Pokfulam Road, Hong Kong, P.R. China
}

\begin{abstract}
We study the problem of reconstructing a super-resolution image $f$ from multiple undersampled, shifted, degraded frames with subpixel displacement errors. The corresponding operator $\mathcal{H}$ is a spatially-variant operator. In this paper, we apply the preconditioned conjugate gradient method with cosine transform preconditioners to solve the discrete problems. Preliminary results show that our method converges very fast and gives sound recovery of the super-resolution images.
\end{abstract}

Keywords: spatially-variant operator, preconditioned conjugate gradient, cosine transform preconditioner, regularization, super-resolution image reconstruction, Neumann boundary condition

\section{INTRODUCTION}

Super-resolution image reconstruction refers to obtaining an image at a resolution higher than that of the camera (sensor) used in recording the image. It has many electronic imaging applications, including aerial or facilities surveillance, consumer, commercial, medical, forensic, and scientific imaging. The observed images often have low resolution. They are also degraded by blur and noise. Increasing the image resolution by using digital signal processing technique $\mathrm{e}^{1,4,5,7,9,10}$ is therefore of great interest.

We consider the reconstruction of a super-resolution image $f$ from multiple undersampled, shifted, degraded and noisy images. Multiple undersampled images are often obtained by using multiple identical image sensors shifted from each other by subpixel displacements. In this application, we usually obtain a spatially-variant system

$$
\mathcal{H} f=g .
$$

Since the system is ill-conditioned and generally not positive definite, we solve it by using minimization and regularization technique:

$$
\min _{f}\|\mathcal{H} f-g\|_{2}^{2}+\alpha \mathcal{R}(f) .
$$

Here $\mathcal{R}(f)$ is a functional which measures the regularity of $f$ and the regularization parameter $\alpha$ is used to control the degree of regularity of the solution. In this paper, we will use the $H_{1}$-norm regularization functional $\|\mathcal{L} f\|_{2}^{2}$ where $\mathcal{L}$ is the first order differential operator.

Previous work (for instance Bose and $\mathrm{Boo}^{1}$ ) pays little attention to the boundary condition of the problem (2). Since we do not have any information about the scene outside the frames, a natural approach is to impose zero boundary conditions outside the scene, i.e. assuming a dark background outside the scene. However, such assumption is usually not satisfied by the images and therefore ringing effect will occur at the boundary of the reconstructed image (see the numerical results in $\mathrm{Ng}$ et $a .^{3,6}$ ). The problem is more severe if the image is reconstructed from a large sensor array. We here use the Neumann boundary condition on the image, which assumes that the scene immediately outside is a reflection of the original scene at the boundary. Our numerical results in $^{3,6}$ have shown that the error of the image under the Neumann boundary condition is less than that under the zero and periodic boundary conditions.

The discretization matrix of $\mathcal{H}$ has a special structure. The preconditioned conjugate gradient (PCG) method is commonly used in solving the system, see Chan and $\mathrm{Ng}^{2}$. We observe that for $2 \times 2$ sensor array with exact subpixel displacement, the matrix system can be solved efficiently by using the discrete cosine transform matrix. We thus propose using the PCG method with cosine transform preconditioners for solving the system when there 
are subpixel displacement errors. Numerical results show that our preconditioners perform significantly better than other preconditioners.

The outline of the paper is as follows. In Section 2, we give a mathematical formulation of the problem. The Neumann boundary condition and a brief introduction on cosine transform preconditioners will be given there. Finally, numerical results are given in Section 3.

\section{SUPER-RESOLUTION IMAGE RECONSTRUCTION}

\subsection{Mathematical Model}

We begin with a brief introduction of the mathematical model in high-resolution image reconstruction. Details can be found in Bose and Boo. ${ }^{1}$

Consider a sensor array with $L_{1} \times L_{2}$ sensors, each sensor has $N_{1} \times N_{2}$ sensing elements (pixels) and the size of each sensing element is $T_{1} \times T_{2}$. Our aim is to reconstruct an image of resolution $M_{1} \times M_{2}$, where

$$
M_{1}=L_{1} \times N_{1} \quad \text { and } \quad M_{2}=L_{2} \times N_{2} .
$$

In order to have some information to resolve the high-resolution image, there are subpixel displacements between the sensors. In the ideal case, the sensors are shifted from each other by a value proportional to $T_{1} / L_{1} \times T_{2} / L_{2}$. However, in practice there can be small perturbations around these ideal subpixel locations due to imperfection of the mechanical imaging system. Thus, for $l_{1}=0,1, \cdots, L_{1}-1$ and and $l_{2}=0,1, \cdots, L_{2}-1$ ( with $\left(l_{1}, l_{2}\right) \neq(0,0)$, the horizontal and vertical displacements $d_{l_{1} l_{2}}^{x}$ and $d_{l_{1} l_{2}}^{y}$ of the $\left[l_{1}, l_{2}\right]$-th sensor array with respect to the $[0,0]$-th reference sensor array are given by

$$
d_{l_{1} l_{2}}^{x}=\frac{T_{1}}{L_{1}}\left(l_{1}+\epsilon_{l_{1} l_{2}}^{x}\right) \quad \text { and } \quad d_{l_{1} l_{2}}^{y}=\frac{T_{2}}{L_{2}}\left(l_{2}+\epsilon_{l_{1} l_{2}}^{y}\right)
$$

Here $\epsilon_{l_{1} l_{2}}^{x}$ and $\epsilon_{l_{1} l_{2}}^{y}$ denote respectively the normalized horizontal and vertical displacement errors. We note that the parameters $\epsilon_{l_{1} l_{2}}^{x}$ and $\epsilon_{l_{1} l_{2}}^{y}$ can be obtained by manufacturers during camera calibration. We assume that $\left|\epsilon_{l_{1} l_{2}}^{x}\right|<\frac{1}{2}$ and $\left|\epsilon_{l_{1} l_{2}}^{y}\right|<\frac{1}{2}$. For if not, the low-resolution images observed from two different sensor arrays will be overlapped so much that the image reconstruction is rendered impossible.

For the super-resolution image reconstruction, we let $S$ be the set of positions of sensors present in the sensor array. We remark that when $S=\left\{\left(l_{1}, l_{2}\right): 0 \leq l_{1} \leq L_{1}-1\right.$, and $\left.0 \leq l_{2} \leq L_{2}-1\right\}$, this just corresponds to the case of the high-resolution image reconstruction. However, when $S \subset\left\{\left(l_{1}, l_{2}\right): 0 \leq l_{1} \leq L_{1}-1\right.$, and $\left.0 \leq l_{2} \leq L_{2}-1\right\}$, this corresponds to the case for the super-resolution image reconstruction.

Let $f$ be the original scene. Then the observed low-resolution image $g_{l_{1} l_{2}}$ for the $\left(l_{1}, l_{2}\right)$-th sensor is modeled by:

$$
g_{l_{1} l_{2}}\left[n_{1}, n_{2}\right]=\int_{T_{2}\left(n_{2}-\frac{1}{2}\right)+d_{l_{1} l_{2}}^{y}}^{T_{2}\left(n_{2}+\frac{1}{2}\right)+d_{l_{1} l_{2}}^{y}} \int_{T_{1}\left(n_{1}-\frac{1}{2}\right)+d_{l_{1} l_{2}}^{x}}^{T_{1}\left(n_{1}+\frac{1}{2}\right)+d_{l_{1} l_{2}}^{x}} f\left(x_{1}, x_{2}\right) d x_{1} d x_{2}+\eta_{l_{1} l_{2}}\left[n_{1}, n_{2}\right],
$$

for $n_{1}=1, \ldots, N_{1}$ and $n_{2}=1, \ldots, N_{2}$. Here $\eta_{l_{1} l_{2}}$ is the noise corresponding to the $\left(l_{1}, l_{2}\right)$-th sensor. We intersperse the low resolution images to form an $L_{1} N_{1} \times L_{2} N_{2}$ image by assigning

$$
g\left[L_{1}\left(n_{1}-1\right)+l_{1}, L_{2}\left(n_{2}-1\right)+l_{2}\right]= \begin{cases}g_{l_{1} l_{2}}\left[n_{1}, n_{2}\right], & \text { if }\left(l_{1}, l_{2}\right) \in S \\ 0, & \text { otherwise }\end{cases}
$$

Here $g$ is an $L_{1} N_{1} \times L_{2} N_{2}$ image and is called the observed image.

Figure 1 shows the method of forming a $4 \times 4$ image $g$ with a $2 \times 2$ sensor array each having a $2 \times 2$ sensing elements, i.e., $L_{1}=2, L_{2}=2, M_{1}=M_{2}=4, N_{1}=N_{2}=2$ and $T_{1}=T_{2}=2$. This is case for the high-resolution image reconstruction and $S=\{(0,0),(0,1),(1,0),(1,1)\}$. We remark that the number of observed image pixel values is the same as the number of unknown original image pixel values. Under the noiseless condition, we have sufficient information (except at the image boundary) to reconstruct the original image exactly. 
However, in the case of the super-resolution image reconstruction, the number of sensors is always less than $L_{1} L_{2}$. For instance, Figure 2 shows a $4 \times 2$ image $g$ with a $2 \times 1$ sensor array each having a $2 \times 2$ sensing elements. Here we consider two sensors, namely, the $[0,0]$-th reference sensor and the $[1,0]$-th sensor, i.e., $S=\{(0,0),(1,0)\}$. This case corresponds that the sensor takes the same scene of the original image but the sensor is slightly displaced in the horizontal direction with respect to the reference sensor. In Figure 3, we consider the $[0,0]$-th reference and $[0,1]$-th sensor arrays. This case corresponds that the sensor is slightly displaced in the vertical direction with respect to the reference sensor, and $S=\{(0,0),(0,1)\}$. In Figure 4, we consider the case of two sensors where the $[1,1]$-th sensor is slightly displaced in the diagonal direction with respect to the $[0,0]$-th reference sensor. In this case, we have $S=\{(0,0),(1,1)\}$

Using a column by column ordering for $g$, we obtain $g=\mathcal{H} f+\eta$ where $\mathcal{H}$ is a spatially variant operator. ${ }^{1}$ Since $\mathcal{H}$ is singular due to the fact that the total number of sensing elements (pixels) is less than the size of the reconstructed image, the classical Tikhonov regularization is used and the minimization problem (2) is solved. In this paper, we use the regularization functionals:

$$
\mathcal{R}(f)=\|\mathcal{L} f\|_{2}^{2}
$$

where $\mathcal{L}$ is the first order differential operator.

\subsection{Image Boundary}

The continuous image model in (3) can be discretized by the rectangular rule and approximated by a discrete image model. Because of the blurring process (cf. (3)), the boundary values of $g$ are also affected by the values of $f$ outside the scene. Thus in solving $f$ from (1), we need some assumptions on the values of $f$ outside the scene. Bose and Boo $^{1}$ imposed the zero boundary condition outside the scene, i.e., assuming a dark background outside the scene in the image reconstruction.

Let $\mathbf{g}$ and $\mathbf{f}$ be respectively the discretization of $g$ and $f$ using a column by column ordering. Under the zero boundary condition, the blurring matrix corresponding to the $\left(l_{1}, l_{2}\right)$-th sensor can be written as

$$
\tilde{\mathbf{H}}_{l_{1} l_{2}}(\epsilon)=\tilde{\mathbf{H}}_{l_{1} l_{2}}^{x}(\epsilon) \otimes \tilde{\mathbf{H}}_{l_{1} l_{2}}^{y}(\epsilon)
$$

where $\tilde{\mathbf{H}}_{l_{1} l_{2}}^{x}(\epsilon)$ is an $M_{1} \times M_{1}$ banded Toeplitz matrix with bandwidth $L+1$ :

$$
\frac{1}{L}\left(\begin{array}{cccccc}
1 & \cdots & 1 & h_{l_{1} l_{2}}^{x+} & & 0 \\
\vdots & \ddots & \ddots & \ddots & \ddots & \\
1 & \ddots & \ddots & \ddots & \ddots & h_{l_{1} l_{2}}^{x+} \\
h_{l_{1} l_{2}}^{x-} & \ddots & \ddots & \ddots & \ddots & 1 \\
& \ddots & \ddots & \ddots & \ddots & \vdots \\
0 & & h_{l_{1} l_{2}}^{x-} & 1 & \cdots & 1
\end{array}\right),
$$

and

$$
h_{l_{1} l_{2}}^{x \pm}=\frac{1}{2} \pm \epsilon_{l_{1} l_{2}}^{x}
$$

The $M_{2} \times M_{2}$ banded blurring matrix $\tilde{\mathbf{H}}_{l_{1} l_{2}}^{y}(\epsilon)$ is defined similarly. We note that ringing effects will occur at the boundary of the reconstructed images if $f$ is indeed not zero close to the boundary, see for instance Figure 5 in $\S 4$. The problem is more severe if the image is reconstructed from a large sensor array since the number of pixel values of the image affected by the sensor array increases.

$\mathrm{Ng}$ et $a .^{6}$ have considered to use the Neumann boundary condition on the image. It assumes that the scene immediately outside is a reflection of the original scene at the boundary. Our numerical results have shown that the Neumann boundary condition gives better reconstructed high resolution images than that by the zero or periodic boundary conditions. Under the Neumann boundary condition, the blurring matrices are still banded matrices with bandwidth $L+1$, but there are entries added to the upper left part and the lower right part of the matrices (see 


\begin{tabular}{|l|l|}
\hline$a_{1}$ & $a_{2}$ \\
\hline$a_{3}$ & $a_{4}$ \\
\hline
\end{tabular}

$g_{00}$

\begin{tabular}{|l|l|}
\hline$c_{1}$ & $c_{2}$ \\
\hline$c_{3}$ & $c_{4}$ \\
\hline
\end{tabular}

$g_{10}$

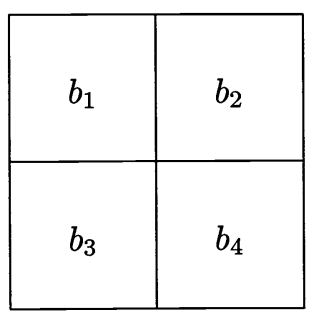

$g_{01}$

\begin{tabular}{|l|l|}
\hline$d_{1}$ & $d_{2}$ \\
\hline$d_{3}$ & $d_{4}$ \\
\hline
\end{tabular}

$g_{11}$

Figure 1: High-resolution image reconstruction.

\begin{tabular}{|l|l|}
\hline$a_{1}$ & $a_{2}$ \\
\hline & $a_{4}$ \\
\hline
\end{tabular}

\begin{tabular}{|l|l|}
\hline$b_{1}$ & $b_{2}$ \\
\hline$b_{3}$ & $b_{4}$ \\
\hline
\end{tabular}

$\longrightarrow \quad$\begin{tabular}{|c|c|c|c|}
\hline$a_{1}$ & $b_{1}$ & $a_{2}$ & $b_{2}$ \\
\hline$*$ & $*$ & $*$ & $*$ \\
\hline$a_{3}$ & $b_{3}$ & $a_{4}$ & $b_{4}$ \\
\hline$*$ & $*$ & $*$ & $*$ \\
\hline
\end{tabular}

$g_{00}$

$g_{01}$

$g$

Figure 2: Super-resolution image reconstruction (horizontal displacement of the sensor).

\begin{tabular}{|l|l|}
\hline$a_{1}$ & $a_{2}$ \\
\hline$a_{3}$ & $a_{4}$ \\
\hline
\end{tabular}

\begin{tabular}{|l|l|}
\hline$c_{1}$ & $c_{2}$ \\
\hline$c_{3}$ & $c_{4}$ \\
\hline
\end{tabular}

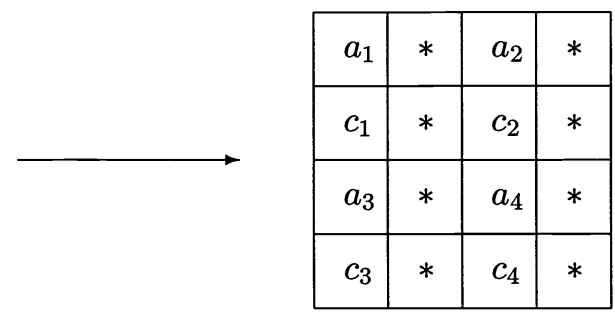

$g_{00}$

$g_{10}$

$g$

Figure 3: Super-resolution image reconstruction (vertical displacement of the sensor).

\begin{tabular}{|l|l|}
\hline$a_{1}$ & $a_{2}$ \\
\hline & \\
$a_{3}$ & $a_{4}$ \\
\hline
\end{tabular}

\begin{tabular}{|l|l|}
\hline$d_{1}$ & $d_{2}$ \\
\hline$d_{3}$ & $d_{4}$ \\
\hline
\end{tabular}

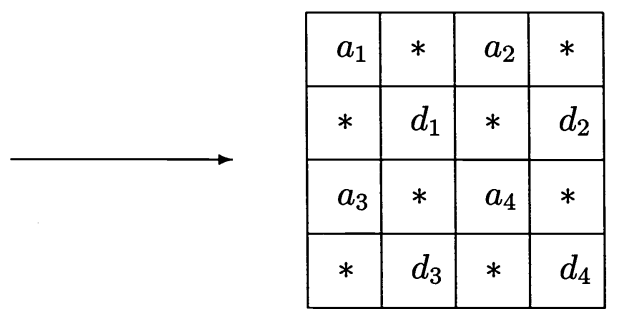

$g_{00}$

$g_{11}$

Figure 4: Super-resolution image reconstruction (diagonal displacement of the sensor). 
the second matrix in (6)). The resulting matrices, denoted by $\mathbf{H}_{l_{1} l_{2}}^{x}(\epsilon)$ and $\mathbf{H}_{l_{1} l_{2}}^{y}(\epsilon)$, have a Toeplitz-plus-Hankel structure:

$$
\frac{1}{L_{1}}\left(\begin{array}{cccccc}
1 & \cdots & 1 & h_{l_{1} l_{2}}^{x+} & & 0 \\
\vdots & \ddots & \ddots & \ddots & \ddots & \\
1 & \ddots & \ddots & \ddots & \ddots & h_{l_{1} l_{2}}^{x+} \\
h_{l_{1} l_{2}}^{x-} & \ddots & \ddots & \ddots & \ddots & 1 \\
& \ddots & \ddots & \ddots & \ddots & \vdots \\
0 & & h_{l_{1} l_{2}}^{x-} & 1 & \cdots & 1
\end{array}\right)+\frac{1}{L_{1}}\left(\begin{array}{cccccc}
1 & \ldots & 1 & h_{l_{1} l_{2}}^{x-} & & \\
\vdots & . & . & & & \\
1 & . \cdot & & & & h_{l_{1} l_{2}}^{x+} \\
h_{l_{1} l_{2}}^{x-} & & & & . & \\
& & & . & . & \vdots \\
0 & & h_{l_{1} l_{2}}^{x+} & 1 & \ldots & 1
\end{array}\right),
$$

and $\mathbf{H}_{l_{1} l_{2}}^{y}(\epsilon)$ is defined similarly. The blurring matrix corresponding to the $\left(l_{1}, l_{2}\right)$-th sensor under the Neumann boundary condition is given by

$$
\mathbf{H}_{l_{1} l_{2}}(\epsilon)=\mathbf{H}_{l_{1} l_{2}}^{x}(\epsilon) \otimes \mathbf{H}_{l_{1} l_{2}}^{y}(\epsilon) .
$$

The blurring matrix for the whole set of sensors is made up of blurring matrices from each sensor:

$$
\mathbf{H}_{S}(\epsilon)=\sum_{\left(l_{1}, l_{2}\right) \in S} \mathbf{D}_{l_{1} l_{2}} \mathbf{H}_{l_{1} l_{2}}(\epsilon) .
$$

Here $\mathbf{D}_{l_{1} l_{2}}$ are diagonal matrices with diagonal elements equal to 1 if the corresponding component of $\mathbf{g}$ comes from the $\left(l_{1}, l_{2}\right)$-th sensor and zero otherwise, see ${ }^{1}$ for more details. More precisely, the matrices $\mathbf{D}_{l_{1} l_{2}}$ are given as follows:

$$
\mathbf{D}_{l_{1} l_{2}}=\left(\mathbf{I}_{M_{1} / L} \otimes \mathbf{D}_{l_{1}}\right) \otimes\left(\mathbf{I}_{M_{2} / L} \otimes \mathbf{D}_{l_{2}}\right),
$$

where $\mathbf{D}_{l}$ are zero $L$-by- $L$ matrices except its $(l+1)$ th main diagonal entry being equal to 1 . For instance, for a $2 \mathrm{x}$ 2 sensor array $(L=2)$,

$$
\mathbf{D}_{0}=\operatorname{diag}(1,0) \text { and } \quad \mathbf{D}_{1}=\operatorname{diag}(0,1) \text {. }
$$

With the Tikhonov regularization, our discretization problem becomes:

$$
\left(\mathbf{H}_{S}(\epsilon)^{t} \mathbf{H}_{S}(\epsilon)+\alpha \mathbf{R}\right) \mathbf{f}=\mathbf{H}_{S}(\epsilon)^{t} \mathbf{g}
$$

where $\mathbf{R}$ is the discretization matrix corresponding to the regularization functional $\mathcal{R}(f)$ in (5). The linear system (8) will be solved by using the conjugate gradient method.

\section{COSINE TRANSFORM PRECONDITIONERS}

Let $\mathbf{C}_{n}$ be the $n \times n$ discrete cosine transform matrix, i.e. the $(i, j)$-th entry of $\mathbf{C}_{n}$ is given by

$$
\sqrt{\frac{2-\delta_{i 1}}{n}} \cos \left(\frac{(i-1)(2 j-1) \pi}{2 n}\right), 1 \leq i, j \leq n,
$$

where $\delta_{i j}$ is the Kronecker delta. Note that the matrix-vector product $\mathbf{C}_{n} x$ can be computed in $O(n \log n)$ operations, see Sorensen and Burrus. ${ }^{8}$ For an $n \times n$ matrix $\mathbf{B}$, the cosine transform preconditioner $c(\mathbf{B})$ of $\mathbf{B}$ is defined to be the matrix $\mathbf{C}_{n}^{t} \Lambda \mathbf{C}_{n}$ that minimizes

$$
\left\|\mathbf{C}_{n}^{t} \Lambda \mathbf{C}_{n}-\mathbf{B}\right\|_{F}
$$

in Frobenius norm. ${ }^{2}$ Clearly, the cost of computing $c(\mathbf{B})^{-1} y$ for any vector $y$ is $O(n \log n)$ operations. For banded matrices, like the one we have in (7), the cost of constructing $c(\mathbf{B})$ is of $O(n)$ only. $^{2}$

When there is no subpixel displacement error, i.e., when $\epsilon_{l_{1} l_{2}}^{x}=\epsilon_{l_{1} l_{2}}^{y}=0$, the matrices $\mathbf{H}_{l_{1}, l_{2}}$ are the same for all $l_{1}$ and $l_{2}$. We will denote them simply by $\mathbf{H}_{L}^{x}$ and $\mathbf{H}_{L}^{y}$. For $L=2$, the blurring matrix $\mathbf{H}_{2}^{x}$ is an $M_{1} \times M_{1}$ tridiagonal matrix given by

$$
\mathbf{H}_{2}^{x}=\frac{1}{2}\left(\begin{array}{ccccc}
\frac{3}{2} & \frac{1}{2} & & & \\
\frac{1}{2} & 1 & \frac{1}{2} & & \\
& \ddots & \ddots & \ddots & \\
& & \frac{1}{2} & 1 & \frac{1}{2} \\
& & & \frac{1}{2} & \frac{3}{2}
\end{array}\right)
$$

and $\mathbf{H}_{2}^{y}$ is an $M_{2} \times M_{2}$ matrix with the same structure. 


\subsection{Horizontal and Vertical Displacement of Sensors}

In this subsection, we consider two sensors in a sensor array where one of the sensors is slightly displaced in the horizontal or vertical direction with respect to an another sensor, i.e., $S=\{(0,0),(1,0)\}$ or $S=\{(0,0),(0,1)\}$ respectively. In these cases, the blurring matrices are given as follows:

$$
\mathbf{H}_{S}(0)^{t} \mathbf{H}_{S}(0)= \begin{cases}\left(\mathbf{H}_{2}^{x} \mathbf{H}_{2}^{x}\right) \otimes \mathbf{H}_{2}^{y}\left(\mathbf{I}_{M_{2} / 2} \otimes \mathbf{D}_{0}\right) \mathbf{H}_{2}^{y}, & \text { when } S=\{(0,0),(1,0)\}, \\ \mathbf{H}_{2}^{x}\left(\mathbf{I}_{M_{1} / 2} \otimes \mathbf{D}_{0}\right) \mathbf{H}_{2}^{x} \otimes\left(\mathbf{H}_{2}^{y} \mathbf{H}_{2}^{y}\right), & \text { when } S=\{(0,0),(0,1)\} .\end{cases}
$$

In this paper, we consider the $H_{1}$-norm regularization functional. Correspondingly, we are required to solve the following linear system:

$$
\left(\mathbf{H}_{S}^{t}(0) \mathbf{H}_{S}(0)+\alpha \mathbf{L} \otimes \mathbf{I}+\alpha \mathbf{I} \otimes \mathbf{L}\right) \mathbf{f}=\mathbf{H}_{S}^{t} \mathbf{g},
$$

where $\alpha>0$ and

$$
\mathbf{L}=\left(\begin{array}{ccccc}
1 & -1 & & & \\
-1 & 2 & -1 & & \\
& \ddots & \ddots & \ddots & \\
& & -1 & 2 & -1 \\
& & & -1 & 1
\end{array}\right)
$$

is the discrete Laplacian matrix with the Neumann boundary condition. The matrix $\mathbf{L}$ can be diagonalized by the discrete cosine transform matrix. It follows that the coefficient matrix $\mathbf{H}_{S}^{t}(0) \mathbf{H}_{S}(0)+\alpha \mathbf{L} \otimes \mathbf{I}_{M_{2}}+\alpha \mathbf{I}_{M_{1}} \otimes \mathbf{L}$ can be transformed to a block-diagonal matrix:

$$
\mathbf{B}_{S}= \begin{cases}\Lambda^{2} \otimes \mathbf{H}_{2}^{y}\left(\mathbf{I}_{M_{2} / 2} \otimes \mathbf{D}_{0}\right) \mathbf{H}_{2}^{y}+\alpha \Gamma \otimes \mathbf{I}_{M_{2}}+\alpha \mathbf{I}_{M_{1}} \otimes \mathbf{L}, & \text { when } S=\{(0,0),(1,0)\}, \\ \mathbf{H}_{2}^{x}\left(\mathbf{I}_{M_{1} / 2} \otimes \mathbf{D}_{0}\right) \mathbf{H}_{2}^{x} \otimes \Lambda^{2}+\alpha \mathbf{L} \otimes \mathbf{I}_{M_{2}}+\alpha \mathbf{I}_{M_{1}} \otimes \Gamma, & \text { when } S=\{(0,0),(0,1)\},\end{cases}
$$

by using $\mathbf{C}_{M_{1}} \otimes \mathbf{I}_{M_{2}}$ or $\mathbf{I}_{M_{1}} \otimes \mathbf{C}_{M_{2}}$ respectively. Here $\Lambda$ and $\Gamma$ are diagonal matrices containing the eigenvalues of $\mathbf{H}_{2}^{x}\left(\right.$ or $\mathbf{H}_{2}^{y}$ ) and $\mathbf{L}$ respectively. We note that the $k$-th diagonal block of $\mathbf{B}_{S}$ is a banded matrix given by

$$
\left[\Lambda^{2}\right]_{k, k} \cdot \mathbf{H}_{2}^{y}\left(\mathbf{I}_{M_{2} / 2} \otimes \mathbf{D}_{0}\right) \mathbf{H}_{2}^{y}+\alpha[\Gamma]_{k, k} \cdot \mathbf{I}_{M_{2}}+\alpha \mathbf{L}
$$

It is easy to see that the bandwidth of each of the diagonal blocks of $\mathbf{B}_{S}$ is equal to 5 and hence its inversion can be done in $O\left(M_{2}\right)$ operations. It follows that the linear system (9) can be solved $4 M_{1}$ one-dimensional fast cosine transforms $\left(2 M_{1}\right.$ for finding the eigenvalues of $\mathbf{H}_{2}^{x}$ and $\mathbf{L}, 2 M_{1}$ for transforming the right hand side and the solution vector). Thus the total cost solving the system is of $O\left(4 M_{1} M_{2} \log M_{2}+M_{1} M_{2}\right)$ operations. Similarly, when $S=\{(0,0),(0,1)\}$, we can show that the total cost of solving the system is of $O\left(4 M_{2} M_{1} \log M_{1}+M_{1} M_{2}\right)$ operations.

\subsection{Spatially-variant Blurring Matrices}

When there are subpixel displacement errors, the blurring matrix $\mathbf{H}_{S}(\epsilon)$ has the same band structure as that of $\mathbf{H}_{S}(0)$, but with some entries slightly perturbed. It can no longer be diagonalized by the cosine transform matrix. Therefore we solve the corresponding linear system by the preconditioned conjugate gradient method. We will use the matrices $\mathbf{H}_{2}^{x}\left(\mathbf{H}_{2}^{y}\right)$ of $\mathbf{H}_{l_{1} l_{2}}^{x}(\epsilon)\left(\mathbf{H}_{l_{1} l_{2}}^{y}(\epsilon)\right)$ as the preconditioner. Thus when $S=\{(0,0),(1,0)\}$, we consider the following preconditioned matrix:

$$
\begin{aligned}
& {\left[\left(\mathbf{H}_{2}^{x} \mathbf{H}_{2}^{x}\right) \otimes \overline{\mathbf{H}}_{l_{1} l_{2}}^{y}(\epsilon)^{t}\left(\mathbf{I}_{M_{2} / 2} \otimes \mathbf{D}_{0}\right) \overline{\mathbf{H}}_{l_{1} l_{2}}^{y}(\epsilon)+\alpha \mathbf{L} \otimes \mathbf{I}_{M_{2}}+\alpha \mathbf{I}_{M_{1}} \otimes \mathbf{L}\right]^{-1} .} \\
& {\left[\mathbf{H}_{00}^{x}(\epsilon)^{t}\left(\mathbf{I}_{M_{1} / 2} \otimes \mathbf{D}_{0}\right) \mathbf{H}_{00}^{x}(\epsilon) \otimes \mathbf{H}_{00}^{y}(\epsilon)^{t}\left(\mathbf{I}_{M_{2} / 2} \otimes \mathbf{D}_{0}\right) \mathbf{H}_{00}^{y}(\epsilon)+\right.} \\
& \left.\mathbf{H}_{10}^{x}(\epsilon)^{t}\left(\mathbf{I}_{M_{1} / 2} \otimes \mathbf{D}_{1}\right) \mathbf{H}_{10}^{x}(\epsilon) \otimes \mathbf{H}_{10}^{y}(\epsilon)^{t}\left(\mathbf{I}_{M_{2} / 2} \otimes \mathbf{D}_{0}\right) \mathbf{H}_{10}^{y}(\epsilon)+\alpha \mathbf{L} \otimes \mathbf{I}_{M_{2}}+\alpha \mathbf{I}_{M_{1}} \otimes \mathbf{L}\right],
\end{aligned}
$$

where

$$
\overline{\mathbf{H}}_{l_{1} l_{2}}^{y}(\epsilon)=\frac{1}{2}\left[\mathbf{H}_{00}^{y}(\epsilon)+\mathbf{H}_{10}^{y}(\epsilon)\right] .
$$

The preconditioned matrix for the case of $S=\{(0,0),(0,1)\}$ can be constructed similarly.

Our numerical results in $\S 3$ show that these preconditioners can indeed speed up the convergence of the method. Since $\mathbf{H}_{S}(\epsilon)$ has at only $(L+1)^{2} / 2$ non-zero diagonals, the matrix-vector product $\mathbf{H}_{L}(\epsilon) \mathbf{x}$ can be done in $O\left(L^{2} M_{1} M_{2}\right)$. Since the linear system involving the preconditioner can be solved in $O\left(M_{1} M_{2} \log M_{1} M_{2}\right)$ operations. Hence the total cost per iteration is of $O\left(M_{1} M_{2} \log M_{1} M_{2}+L^{2} M_{1} M_{2}\right)$ operations. 


\subsection{Diagonal Displacement of Sensors}

In this subsection, we also consider two sensors in a sensor array. However, one of the sensors is slightly displaced in the diagonal direction with respect to an another sensor, i.e., $S=\{(0,0),(1,1)\}$. In this case, we solve the following linear system:

$$
\begin{aligned}
& \left\{\left[\mathbf{H}_{00}^{x}(\epsilon)^{t}\left(\mathbf{I}_{M_{1} / 2} \otimes \mathbf{D}_{0}\right) \mathbf{H}_{00}^{x}(\epsilon)\right] \otimes\left[\mathbf{H}_{00}^{y}(\epsilon)^{t}\left(\mathbf{I}_{M_{2} / 2} \otimes \mathbf{D}_{0}\right) \mathbf{H}_{00}^{y}(\epsilon)\right]+\right. \\
& {\left.\left.\left[\mathbf{H}_{11}^{x}(\epsilon)^{t}\left(\mathbf{I}_{M_{1} / 2} \otimes \mathbf{D}_{1}\right) \mathbf{H}_{11}^{x}(\epsilon)\right] \otimes\left[\mathbf{H}_{11}^{y}(\epsilon)^{t}\left(\mathbf{I}_{M_{2} / 2} \otimes \mathbf{D}_{1}\right) \mathbf{H}_{11}^{y}(\epsilon)\right]+\alpha \mathbf{L} \otimes \mathbf{I}_{M_{2}}+\alpha \mathbf{I}_{M_{1}} \otimes \mathbf{L}\right]\right\} \mathbf{f} } \\
= & \left\{\left[\mathbf{H}_{00}^{x}(\epsilon)^{t}\left(\mathbf{I}_{M_{1} / 2} \otimes \mathbf{D}_{0}\right)\right] \otimes\left[\mathbf{H}_{00}^{y}(\epsilon)^{t}\left(\mathbf{I}_{M_{2} / 2} \otimes \mathbf{D}_{0}\right)\right]+\left[\mathbf{H}_{11}^{x}(\epsilon)^{t}\left(\mathbf{I}_{M_{1} / 2} \otimes \mathbf{D}_{1}\right)\right] \otimes\left[\mathbf{H}_{11}^{y}(\epsilon)^{t}\left(\mathbf{I}_{M_{2} / 2} \otimes \mathbf{D}_{1}\right)\right]\right\} \mathbf{g}
\end{aligned}
$$

We employ the coefficient matrix arising from the case of horizontal or vertical displacement of sensors as a preconditioner. More precisely, we have the following two preconditioners for the blurring matrix in (11) :

$$
\frac{1}{2}\left\{\mathbf{H}_{2}^{x} \mathbf{H}_{2}^{x} \otimes\left[\mathbf{H}_{00}^{y}(\epsilon)^{t}\left(\mathbf{I}_{M_{2} / 2} \otimes \mathbf{D}_{0}\right) \mathbf{H}_{00}^{y}(\epsilon)\right]+\mathbf{H}_{2}^{x} \mathbf{H}_{2}^{x} \otimes\left[\mathbf{H}_{11}^{y}(\epsilon)^{t}\left(\mathbf{I}_{M_{2} / 2} \otimes \mathbf{D}_{1}\right) \mathbf{H}_{11}^{y}(\epsilon)\right]\right\}+\alpha \mathbf{L} \otimes \mathbf{I}_{M_{2}}+\alpha \mathbf{I}_{M_{1}} \otimes \mathbf{L}
$$

and

$$
\frac{1}{2}\left\{\left[\mathbf{H}_{00}^{x}(\epsilon)^{t}\left(\mathbf{I}_{M_{1} / 2} \otimes \mathbf{D}_{0}\right) \mathbf{H}_{00}^{x}(\epsilon)\right] \otimes \mathbf{H}_{2}^{y} \mathbf{H}_{2}^{y}+\left[\mathbf{H}_{11}^{x}(\epsilon)^{t}\left(\mathbf{I}_{M_{1} / 2} \otimes \mathbf{D}_{1}\right) \mathbf{H}_{11}^{x}(\epsilon)\right] \otimes \mathbf{H}_{2}^{y} \mathbf{H}_{2}^{y}\right\}+\alpha \mathbf{L} \otimes \mathbf{I}_{M_{2}}+\alpha \mathbf{I}_{M_{1}} \otimes \mathbf{L} .
$$

Our numerical results in $\S 3$ show that these preconditioners are quite effective. For the cost of using these two preconditioners, we can transform them to a block-diagonal matrix and each diagonal block is just a banded matrix. Thus the total cost of solving the system involving the preconditioner is of $O\left(M_{1} M_{2} \log M_{2}+M_{1} M_{2}\right)$ operations. Hence, the total cost per iteration is again of $O\left(M_{1} M_{2} \log M_{1} M_{2}+L^{2} M_{1} M_{2}\right)$ operations.

\section{NUMERICAL EXAMPLES}

In this section, we illustrate the effectiveness of preconditioned conjugate gradient method by solving the superresolution image reconstruction problem with a $2 \times 2$ sensor array. The original $128 \times 128$ image is shown in Figure $5(\mathrm{a})$. In the tests, we use the zero vector as the initial guess in the conjugate gradient method. The stopping criteria is $\left\|\mathbf{r}^{(j)}\right\|_{2} /\left\|\mathbf{r}^{(0)}\right\|_{2}<10^{-6}$, where $\mathbf{r}^{(j)}$ is the normal equations residual after $j$ iterations.

Firstly, the parameters $\epsilon_{l_{1} l_{2}}^{x}$ and $\epsilon_{l_{1} l_{2}}^{y}$ are set to be zero. We show the $128 \times 128$ reconstructed images from (i) four $64 \times 64$ low resolution images, i.e., the high-resolution image reconstruction case and (ii) two $64 \times 64$ low-resolution images, i.e., the super-resolution image reconstruction case. One of the low-resolution images is shown in Figure 5 (b). Gaussian white noises with signal-to-noise ratios of $50 \mathrm{~dB}$ and $30 \mathrm{~dB}$ are added to the low-resolution images. The observed high-resolution noisy images $\mathbf{g}$ are shown as in Figures 5(c) (high-resolution case), 5(e) $(\rightarrow), 5(\mathrm{~g})$ $(\downarrow), 5(\mathrm{i})(\searrow)$ and 6(c) (high-resolution case), 6(e) $(\rightarrow), 6(\mathrm{~g})(\downarrow), 6(\mathrm{i})(\searrow)$. Here $\rightarrow, \downarrow$ and $\searrow$ corresponds that the second sensor is slightly displaced in the horizontal, vertical and diagonal directions with respect to the first sensor respectively. Figures $5(\mathrm{~d}), 5(\mathrm{f}), 5(\mathrm{~h}), 5(\mathrm{j})$ and $6(\mathrm{~d}), 6(\mathrm{f}), 6(\mathrm{~h}), 6(\mathrm{j})$ show their corresponding reconstructed images. For Figures 5(c) and 6(c), the images are reconstructed by using four $64 \times 64$ low resolution images. For Figures $5(\mathrm{e}), 5(\mathrm{~g}), 5(\mathrm{i}), 6(\mathrm{e}), 6(\mathrm{~g})$ and $6(\mathrm{i})$, the images are reconstructed by using two $64 \times 64$ low resolution images. The optimal regularization parameter $\alpha$ is chosen such that it minimizes the relative error of the reconstructed image $\mathbf{f}_{r}(\alpha)$ to the original image $\mathbf{f}$, i.e., it minimizes

$$
\frac{\left\|\mathbf{f}-\mathbf{f}_{r}(\alpha)\right\|_{2}}{\|\mathbf{f}\|_{2}}
$$

We see that the trees in the image are much better reconstructed under the super-resolution case (cf. Figures 5 (e), $5(\mathrm{~g}), 5(\mathrm{i}), 6(\mathrm{e}), 6(\mathrm{~g})$ and 6(i)) than the high-resolution observed images. (cf. Figures 5(b), 5(c), 6(b) and 6(c)). We remark that the relative errors of the super-resolution reconstructed images (cf. Table 1) are less than the relative errors of the high-resolution observed images are 0.0758 and 0.0765 for SNR $=50 \mathrm{~dB}$ and $30 \mathrm{~dB}$ respectively.

Table 1 shows the optimal regularization parameters and the corresponding relative errors. We note from the table that the number of iterations required for convergence is less when the SNR is high. Both the relative errors and the numbers of iterations are low when the second sensor is slightly displaced in the diagonal direction with respect to the first sensor. This phenomenon may be explained that the horizontal and vertical details may be more 
(a) Original Image

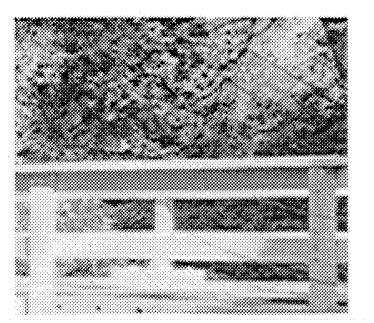

(c) Observed high-resolution image

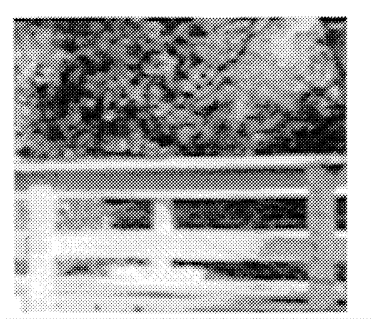

(e) Observed super-resoldion imace

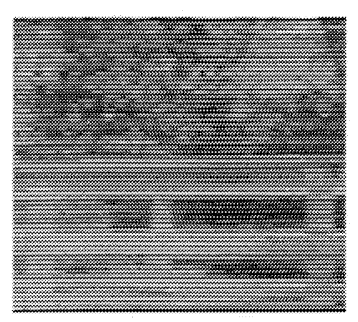

(g) Observed super-resoldion image

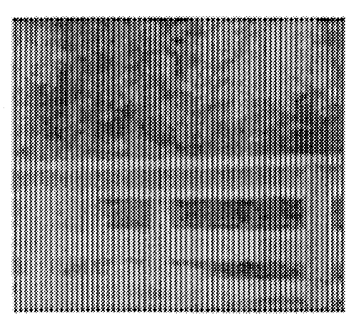

(1) Oossenved super-resolution image

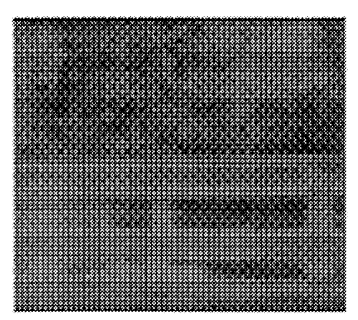

(b) Low-resolution image

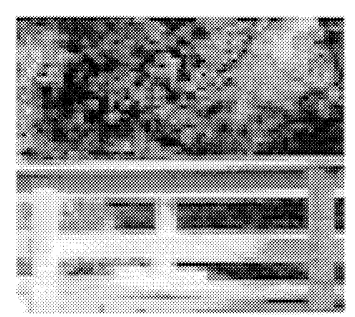

(o) Reconstructed high-resoldion image

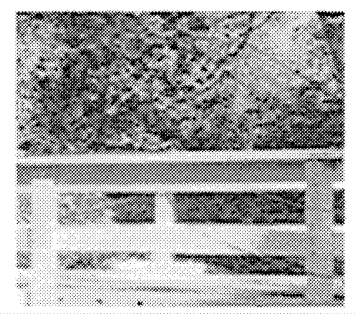

(i) Reconstructed Super-resoldtion image

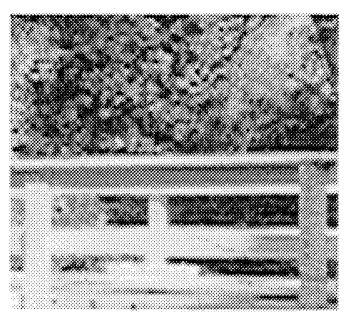

(h) Reconsinuded super-resolution imaage

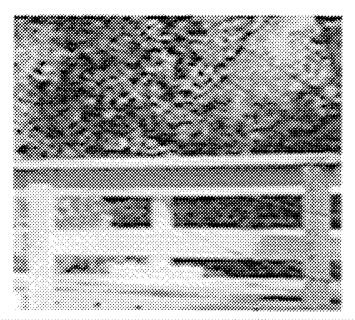

(1) Reconsincueded super-resolution image

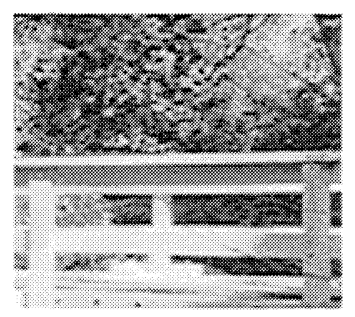

(a) Original imaage

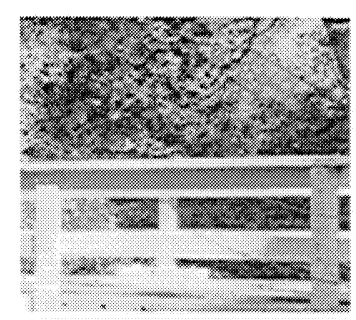

(c) Obsenved high-resolution imaage

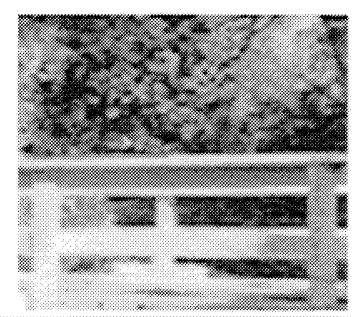

(e) Observed super-resolution image

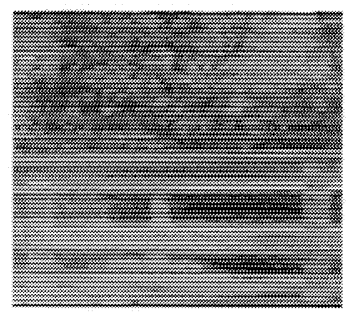

(g) Obsenved super-resolution image

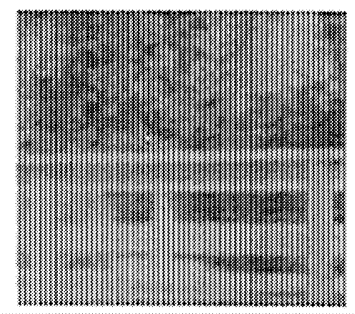

(1) Ohserved super-resolution image

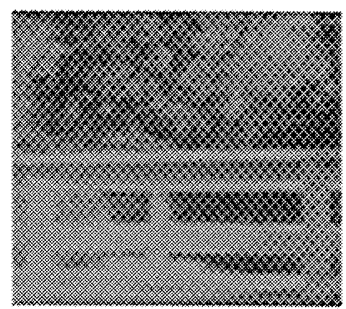

(b) Low-resolution image

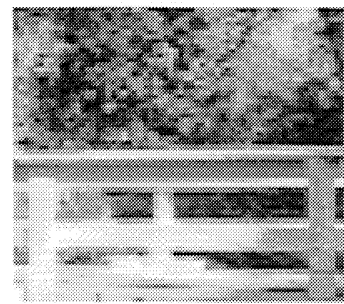

(d) Reconsisucted hich-resoldion image

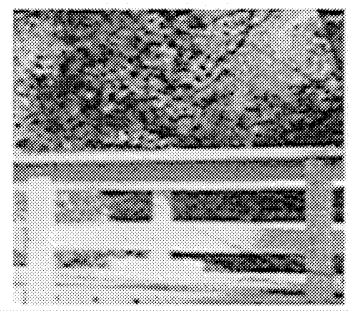

(i) Reconstucheds super-resolution image

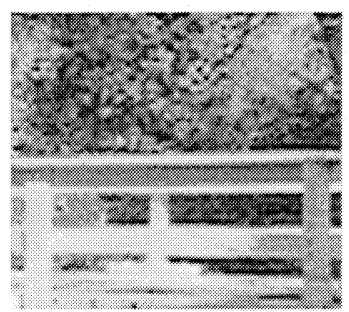

(h) Reconsinucted super-resolution image

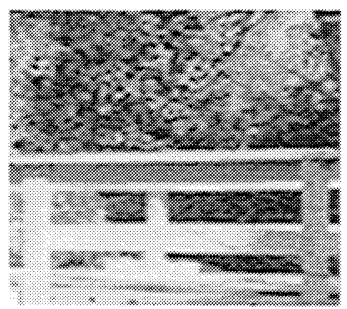

(i) Reconstructed super-resolution image

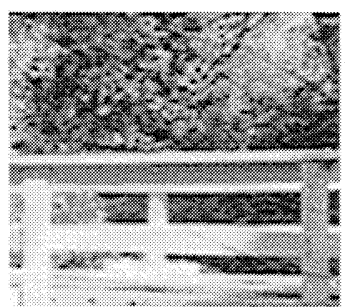

Figure 5. (left two columns) Images for high- and super-resolution cases when SNR $=50 \mathrm{~dB}$, and Figure 6. (right two columns) Images for high- and super-resolution cases when SNR $=30 \mathrm{~dB}$ (right). 


\begin{tabular}{|c|c|c|c|c|c|}
\hline$S$ & $\alpha$ & Relative error & $S$ & $\alpha$ & Relative error \\
\hline$\{(0,0),(0,1),(1,0),(1,1)\}$ & $5 \times 10^{-5}$ & 0.0366 & $\{(0,0),(0,1),(1,0),(1,1)\}$ & $5 \times 10^{-3}$ & 0.0558 \\
\hline$\{(0,0),(0,1)\} \rightarrow$ & $5 \times 10^{-5}$ & 0.0703 & $\{(0,0),(0,1)\} \rightarrow$ & $5 \times 10^{-3}$ & 0.0761 \\
\hline$\{(0,0),(1,0)\}$ & $5 \times 10^{-5}$ & 0.0622 & $\{(0,0),(1,0)\} \downarrow$ & $5 \times 10^{-3}$ & 0.0703 \\
\hline$\{(0,0),(1,1)\}$ & $5 \times 10^{-5}$ & 0.0533 & $\{(0,0),(1,1)\} \searrow$ & $5 \times 10^{-3}$ & 0.0630 \\
\hline
\end{tabular}

Table 1. Regularization parameters and relative errors when SNRs are $50 \mathrm{~dB}$ (left) and $30 \mathrm{~dB}$ (right).

\begin{tabular}{c|ccc|ccc}
$M_{1}=M_{2}$ & Without preconditioner & Cosine & & $M_{1}=M_{2}$ & Without preconditioner & Cosine \\
\hline 32 & 232 & 12 & & 32 & 39 & 5 \\
64 & 257 & 12 & & 64 & 42 & 5 \\
128 & 250 & 11 & & 128 & 40 & 5 \\
256 & 251 & 11 & & 256 & 40 & 5
\end{tabular}

Table 2. Number of iterations required for convergence when SNRs are $50 \mathrm{~dB}$ (left) and $30 \mathrm{~dB}$ (right).

\begin{tabular}{c|ccc|ccc}
$M_{1}=M_{2}$ & Without preconditioner & Cosine & & $M_{1}=M_{2}$ & Without preconditioner & Cosine \\
\hline 32 & 148 & 80 & & 32 & 27 & 14 \\
64 & 156 & 87 & & 64 & 27 & 14 \\
128 & 134 & 84 & & 128 & 26 & 13 \\
256 & 132 & 72 & & 256 & 26 & 13
\end{tabular}

Table 3. Number of iterations required for convergence when SNRs are $50 \mathrm{~dB}$ (left) and $30 \mathrm{~dB}$ (right).

accurately interpolated under the diagonal sensor displacement setting than under the horizontal and vertical sensor displacement setting.

Tables 2 and 3 show the performance of our cosine transform based preconditioners proposed in Section 2. Here we consider two sensors. One of sensors is slightly displaced in the horizontal direction (Table 2), and is slightly displaced in the diagonal direction (Table 3). In the tables, $\alpha$ is chosen to be $10^{-5}$, and the parameters $\epsilon_{l_{1} l_{2}}^{x}$ and $\epsilon_{l_{1} l_{2}}^{y}$ are chosen randomly between -0.1 and 0.1 . We see from Table 2 that the cosine transform preconditioner converges significantly faster than without using the preconditioner. To further illustrate the fast convergence of our method, Figure 6 depicts the convergence behaviour of the original system and preconditioned system when $M_{1}=M_{2}=128$. The figure shows that the preconditioned system converges very quickly. Although we find that the cosine transform preconditioner converges faster than without using the preconditioner in Table 3, the performance of using the cosine transform preconditioner is not very superior to that without using the preconditioner. Again Figure 7 depicts the convergence behaviour of the original system and preconditioned system when $M_{1}=M_{2}=128$. We see from the figure that the convergence of the preconditioned system in the initial few iterations is very fast and then the convergence slows down.

\section{ACKNOWLEDGMENTS}

The work of Michael Ng was supported by Hong Kong Research Grants Council Grant No. HKU 7147/99P and HKU CRCG Grant No. 10202720.

\section{REFERENCES}

1. N. K. Bose and K. J. Boo. "High-resolution image reconstruction with multisensors", International Journal of Imaging Systems and Technology, 9 (1998), pp. 294-304.

2. R. H. Chan and M. K. Ng. "Conjugate gradient method for Toeplitz system", SIAM Review, 38, pp. 427-482, 1996. 

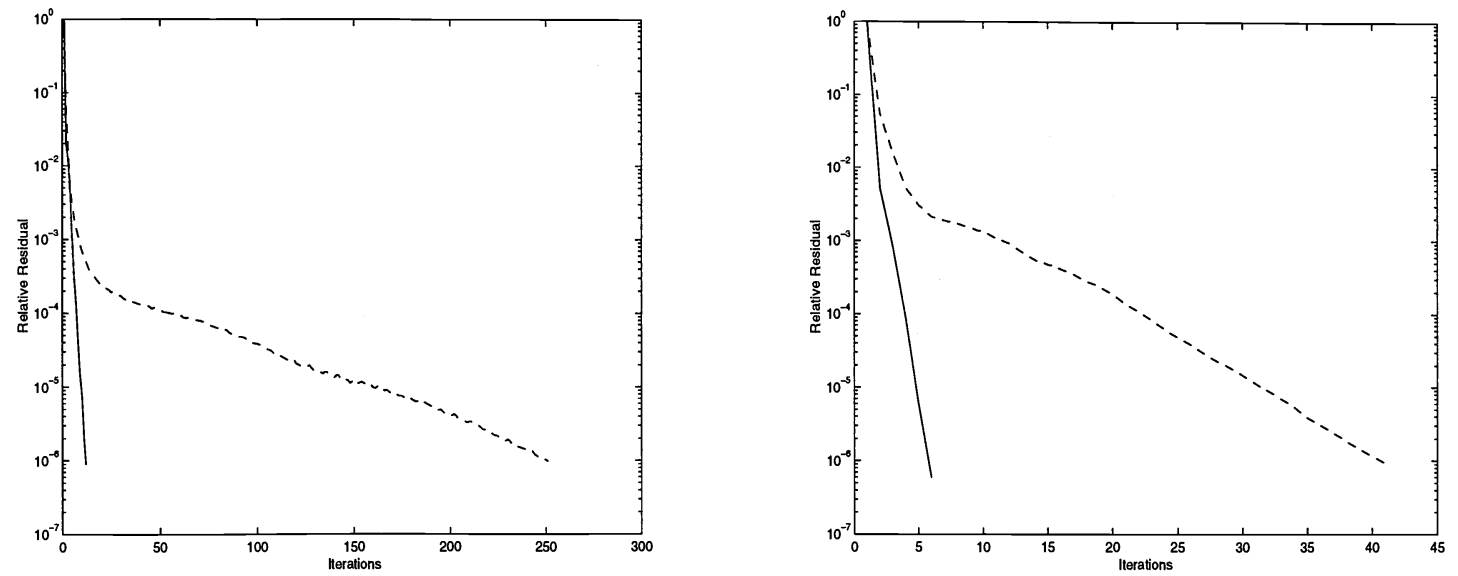

Figure 6. Convergence behaviour of the original system (- - ) and the cosine transform preconditioned system (-) when SNRs are $50 \mathrm{~dB}$ (left) and $30 \mathrm{~dB}$ (right).
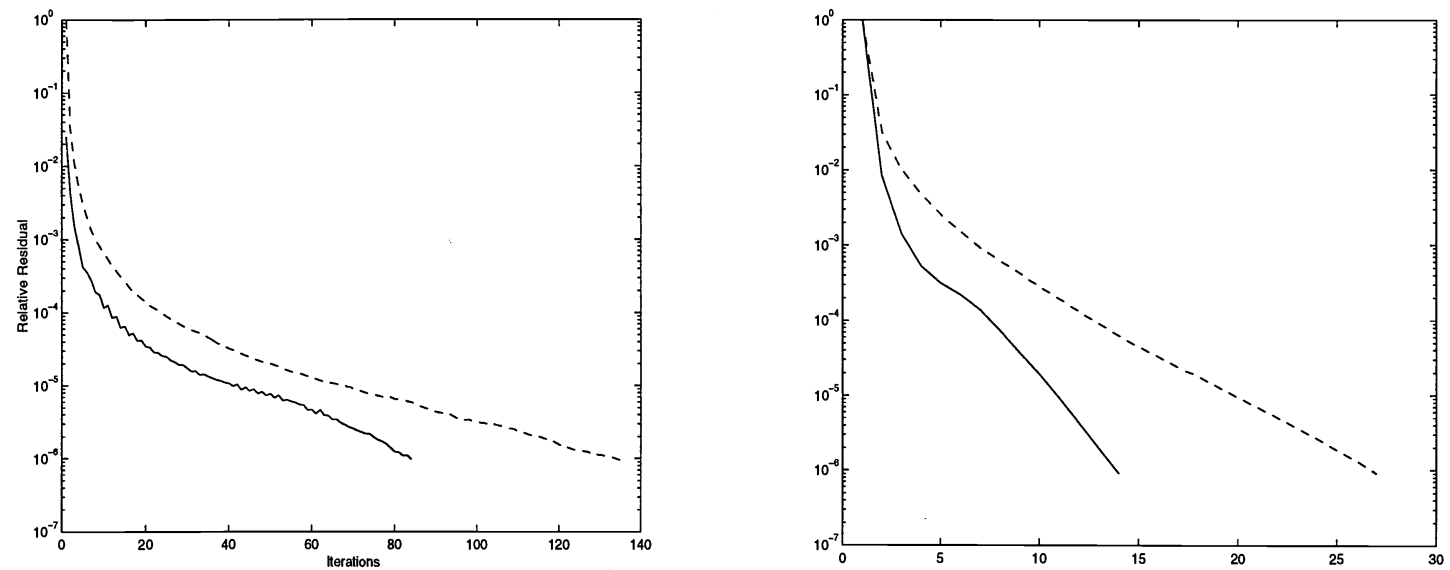

Figure 7. Convergence behaviour of the original system (- - ) and the cosine transform preconditioned system (-) when SNRs are 50dB (left) and 30dB (right).

3. R. Chan, T. Chan, M. Ng, W. Tang, and C. Wong, Preconditioned iterative methods for high-resolution image reconstruction with multisensors, Proceedings to the SPIE Symposium on Advanced Signal Processing: Algorithms, Architectures, and Implementations, Vol. 3461, San Diego CA, July, 1998, Ed: F. Luk.

4. E. Kaltenbacher and R. C. Hardie. "High resolution infrared image reconstruction using multiple, low resolution, aliased frames", Proc. of IEEE 1996 national aerospace and electronic conf. NAECON 2, pp. 702-709, 1996.

5. S. P. Kim, N. K. Bose and H. M. Valenzuela. "Recursive reconstruction of high resolution image from noisy undersampled multiframes", IEEE Trans. on Acoust., Speech, and Signal Process, 38(6), pp. 1013-1027, 1990.

6. M. Ng. R. Chan, T. Chan and A. Yip, Cosine transform preconditioners for high resolution image reconstruction, Res. Rept. 99-10, Dept. Math., The Chinese University of Hong Kong, or Linear Algebra Appls., to appear.

7. R. R. Schultz and R. L Stevenson. "Extraction of high-resolution frames from video sequences", IEEE T. Image Proces., 5(6), pp. 996-1011, 1996.

8. H. Sorensen and C. Burrus, "Fast DFT and convolution algorithms", Handbook of Signal Processing, edited by S. Mitra and J. Kaiser, New York, Wiley.

9. A. M. Tekalp, M. K. Ozkan and M. I. Sezan, "High-resolution image reconstruction from lower-resolution image sequences and space-varying image restoration", In Proc. IEEE Int. Conf. Acoust., Speech, and Signal Process, III, pp. 169-172, San Francisco, CA, March 1992.

10. R. Y. Tsai and T. S. Huang. "Multiframe image restoration and registration", Advances in Computer Vision and Image Processing, 1, pp. 317-339, 1984. 